\title{
Visualization and monitoring of damaging-healing processes of polymers by AIEgen- loaded multifunctional microcapsules
}

Shusheng Chen, Ting Han*, Junkai Liu, Xinting Liang, Jinglei Yang*, and Ben Zhong Tang*

Dr. S. Chen, Dr. T. Han

Center for AIE Research, Shenzhen Key Laboratory of Polymer Science and Technology, Guangdong Research Center for Interfacial Engineering of Functional Materials, College of Materials Science and Engineering, Shenzhen University, Shenzhen 518060, China

E-mail: hanting@szu.edu.cn

Dr. S. Chen, Prof. J. Yang

Department of Mechanical and Aerospace Engineering, The Hong Kong University of Science and Technology, Clear Water Bay, Hong Kong SAR, China; HKUST Shenzhen-Hong Kong Collaborative Innovation Research Institute, Futian, Shenzhen 518048, China

E-mail: maeyang@ust.hk

Dr. J. Liu, Prof. B. Z. Tang

Department of Chemistry, Hong Kong Branch of Chinese National Engineering Research Center for Tissue Restoration and Reconstruction, The Hong Kong University of Science and Technology, Clear Water Bay, Kowloon, Hong Kong, China

Dr. X. Liang

Key Laboratory of Bioorganic Chemistry and Molecular Engineering of Ministry of Education and Beijing National Laboratory for Molecular Science, and Peking-Tsinghua Center for Life Sciences, Peking University, Beijing, 100871, China.

Prof. B. Z. Tang

Shenzhen Institute of Aggregate Science and Technology, School of Science and Engineering, The Chinese University of Hong Kong, Shenzhen, 2001 Longxiang Boulevard, Longgang District, Shenzhen City, Guangdong 518172, China

E-mail: tangbenz@cuhk.edu.cn

Keywords: Aggregation-induced emission, Process visualization, Damaging-healing process, Microcapsule 


\section{Abstract:}

Polymeric materials play an essential and ubiquitous role in modern societies, but they are inevitably damaged during service, which can lead to compromised performance or even direct failure. The sensitive detection and dynamic monitoring of the health states of polymers is thus crucial to increase their reliability, safety, and lifetime. Herein, a facile fluorescence-based approach that can achieve the nondestructive, on-site, real-time, full-field, and sensitive visualization and monitoring of damaging-healing processes of polymers is demonstrated. By embedding novel UV-blocking microcapsules containing a diisocyanate solution of aggregation-induced emission luminogens (AIEgens) into a polymer matrix, the damaged regions of the composite show turn-on fluorescence and dual signal changes in both fluorescence intensity and fluorescence color can be observed during the healing processes. The invisible information of the static health states and dynamic healing processes can be directly and semi-quantitatively visualized by naked eyes based on the collective effects of AIE and twisted intramolecular charge transfer. In addition to the autonomous damage-reporting, self-healing, and health indication functionalities, the microcapsule-embedded polymeric coatings possess excellent photo- and water-protection capabilities, which are appealing to various practical applications.

\section{Introduction}

Polymers are everywhere in our daily life and play an indispensable role in the development of modern societies. They are widely used as structural materials or functional coatings in housewares, biomedical supplies, aerospace industry, marine transportation, electronic packaging, etc. Polymers are required to be as robust as possible for most engineering applications. However, damage, especially small-scale cracks that results from manufacture processes or environmental stress during usage, is inevitable. ${ }^{[1]}$ The occurrence and 
propagation of such small-scale damage can cause the compromise of structural integrality of polymers and eventually lead to catastrophic failure in materials performance. For example, the existence of microcracks in polymer parts of aircraft could grow into macroscopic damage under certain circumstances, which may cause the risk of serious aviation disasters. ${ }^{[2]}$ In marine industries, the corrosion resistance of polymeric coatings will be greatly compromised once micro- or mesoscopic cracks occur, consequently causing significant environmental and economic losses. ${ }^{[3]}$ Additionally, microcracks in functional polymer coatings for the packaging of precise electronics are harmful for their protection properties such as vacuum sealing, waterproof and lightproof performances, ultimately resulting in the performance failure of the electronic products. ${ }^{[4]}$ Therefore, to improve the quality, reliability, and durability of critical polymeric components, it is crucial to sensitively detect and monitor damage in polymers at an early stage and meanwhile autonomously repair the damaged regions before malfunction occurs. ${ }^{[5-6]}$

Conventional techniques for detecting mechanical damage or evaluating the structural health of polymers usually relied on optical and electron microscopies, such as metallurgical microscopy, scanning electron microscopy (SEM), and transmission electron microscopy. ${ }^{[7-8]}$ Although these techniques have been widely used, their sample preparation procedures are often troublesome and may cause an irreversible change in the sample structure. In some cases, non-destructive methods including infrared imaging, radiography, and ultrasonic imaging were utilized to detect the health states of critical structural components. ${ }^{[9]}$ However, these methods are generally time-consuming and require high-cost instruments and equipment. Well-trained technicians are often needed to operate the instruments and analyze the indirect output signals or curves. Moreover, the abovementioned methods are not suitable for the in situ and real-time detection and monitoring of damage processes. ${ }^{[10]}$ To effectively expand the life span of polymers, diverse smart polymer composites with self-healing capability have been 
developed. ${ }^{[11-12]}$ However, their healing degree and the dynamic healing processes are also difficult to be sensitively and directly visualized through traditional characterization methods. As seeing is believing, the development of simple and direct methods that can achieve the realtime, on-site, full-field, and sensitive visualization and monitoring of damaging-healing processes of polymers is thus highly desirable in both academia and industry.

Fluorescence-based methods are the most promising candidates due to their advantages of visible and portable detection, fast response, non-invasiveness, high sensitivity, and large contrast. ${ }^{[13-14]}$ Nevertheless, conventional fluorophores are usually non-luminescent or weakly emissive in condensed states due to the notorious aggregation-caused quenching effect, ${ }^{[15]}$ which greatly limits their applications as optical indicators in the solid-state detection of damage in polymeric materials. Moreover, the poor photobleaching resistance of such fluorophores also hinders their applications in the real-time monitoring of dynamic processes. Fortunately, aggregation-induced emission luminogens (AIEgens) provide a good solution to this problem. ${ }^{[16-21]}$ AIEgens show weak or no fluorescence in solutions but become highly emissive upon aggregation. A well-accepted working mechanism for AIEgens is the restriction of intramolecular motions (RIM). Theoretically speaking, the fluorescence of AIEgens can be lightened up or enhanced in any microenvironment that can restrict their intramolecular motions. The bright solid-state fluorescence and excellent photostability of AIEgens as well as their sensitive response to microenvironment variation enable them to serve as ideal optical indicators to visualize both the static health states and the dynamic damaging-healing processes with low background noise. Attracted by the unique features of AIEgens, great efforts have been devoted to applying AIEgens for the damage visualization in polymers. ${ }^{[22-23]}$ For example, Moore et al. demonstrated a robust and versatile design for the turn-on detection of damage in polymeric materials by embedding AIEgen-loaded microcapsules (MCs) into matrices. ${ }^{[24]}$ This turn-on fluorescence system was further extended to a ratiometric system that can respond to 
mechanical deformation ${ }^{[25-26]}$ and a multilayered system that can report the penetration depth of cracks $^{[27]}$ via the change in fluorescence color. Recently, smart polymer coatings with damage-reporting and healing dual functions were also developed by simultaneously encapsulating AIEgens and healing agents in microcapsules. ${ }^{[28-29]}$ Park et al. utilized a fluorophore with different fluorescence colors in solution and solid state to indicate the static damaged and healed state of polymers. ${ }^{[30-31]}$ However, the MC composition and the coating structure in this UV-induced healing system are complicated for practical applications, and the mechanistic insights need to be further investigated to provide a clear design guideline.

Herein, we aim to develop a simple yet multifunctional AIEgen-based MC-polymer composite system with sensitive damage self-reporting capability and spontaneous self-healing function. To increase the evaluation accuracy of damage and meanwhile to achieve the visual monitoring of the dynamic self-healing reaction in damaged regions, the single-component MCs are designed to provide dual signal changes in both fluorescence intensity and fluorescence color. Furthermore, instead of modifying coating structures, MCs with novel and multifunctional shells will be prepared. The incorporation of UV-blocking shells can not only enhance the sensitivity and contrast of detection but also provide photoprotection for the sensitive core.

To achieve the dual-signal indication, we first proposed a possible design concept based on the working mechanism of AIEgen with twisted intramolecular charge transfer (TICT) effects (referred as TICT-type AIEgen). ${ }^{[32-33]}$ As schematically illustrated in Figure 1a, owing to the twisted donor (D)-acceptor (A) structures, both the fluorescence intensity and fluorescence color of TICT-type AIEgen can show sensitive response to their surrounding microenvironments such as rigidity and polarity. ${ }^{[34-36]}$ TICT-type AIEgen is capable of showing obvious fluorescence in solvents with suitable polarity. With an increase in the restriction effect or a decrease in the polarity of microenvironments, the TICT-type AIEgen would show an 
obvious blueshift in the emission color accompanied with remarkably enhanced emission intensity due to the collective influences of TICT and RIM. In the free or polar environments such as in relatively polar solvents, the TICT-type AIEgen will be in the TICT state with its D and A units existing in a twisted conformation. The total charge separation of TICT state leads to a relatively narrower bandgap than the locally excited (LE) state. When the intramolecular motions of TICT-type AIEgen are restricted by solidification, its fluorescence intensity will be gradually enhanced due to the AIE effect. Meanwhile, the D and A units might adopt an almost parallel arrangement in the excited state to show the LE-state fluorescence with a blue-shifted emission wavelength.

Taking advantage of this unique characteristic of TICT-type AIEgen, a facile design to visualize the entire damaging-healing processes of MC-based self-healing polymers was illustrated in Figure 1b. Diisocyanate, which automatically reacts with moisture, is an ideal liquid core that can function as both the solvent of TICT-type AIEgen and the inherent healing agent. $^{[37-38]}$ The diisocyanate solution of a TICT-type AIEgen is first encapsulated by novel UV-blocking shells to form robust and potentially UV-blocking MCs. Then the AIEgen-loaded functional MCs will be dispersed in polymer matrix. When the MC-embedded polymer coating is damaged, the broken MCs will release the diisocyanate solution of TICT-type AIEgen into the cracks to show obvious fluorescence in the damaged regions. Meanwhile, the released diisocyanate would gradually change from liquid state to solid state because of its spontaneous reaction with moisture in air. During this healing polymerization, the damaged regions are expected to exhibit blue-shifted emission color and increased emission intensity due to the increasing restriction of the TICT-type AIEgen. In this sense, both the static health states and the whole damaging-healing processes of the MC-based autonomous polymer coatings will be facilely and directly visualized by the dual-signal indication in an in-situ and real-time way. 
Combined with the potential light blocking properties of the MCs, the MC-embedded polymer composites could be developed as multifunctional coating materials.

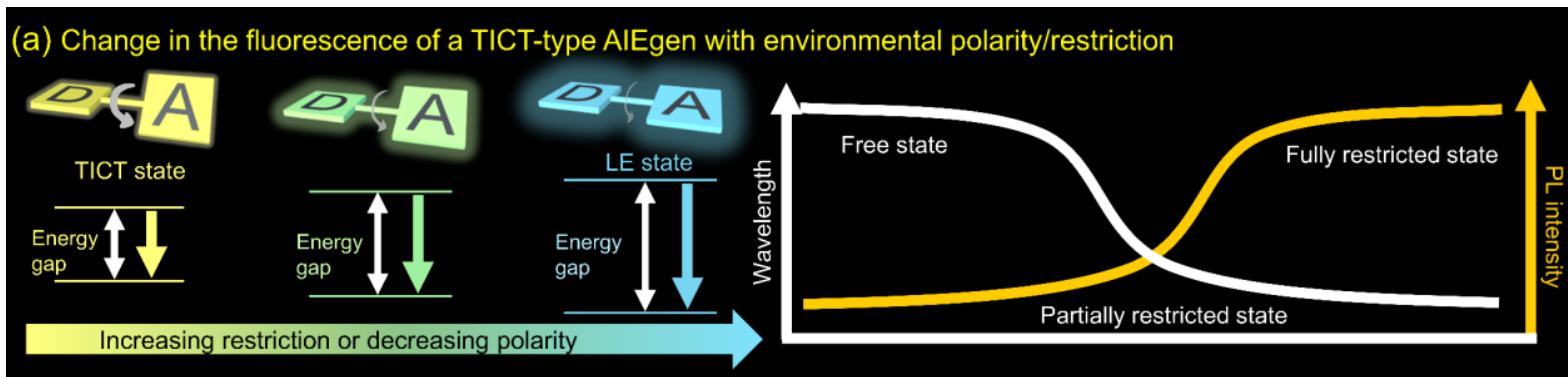

(b) Application in the visualization of health state of polymer coating

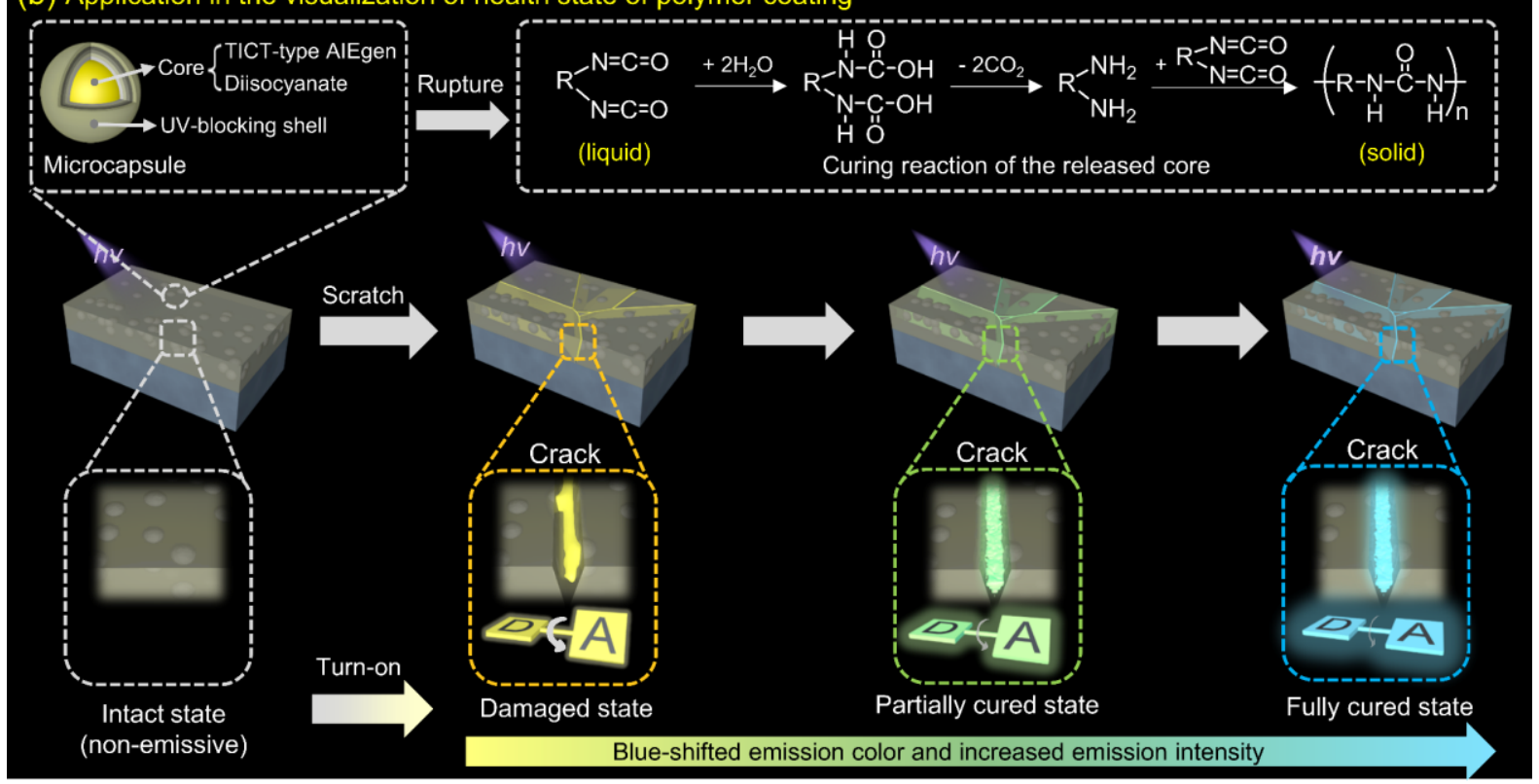

Figure 1. Schematic illustration of the design principle. (a) Fluorescence change of TICT-type AIEgen with environmental polarity/restriction and (b) Working mechanism of microcapsules containing TICT-type AIEgen and isocyanate for the visualization of health states of polymer coatings.

To verify the feasibility of the design principle, three TICT-type AIEgens with typical D- $\pi$ A structures named as DMTPE-BMO, TPA-BMO, and TPE-BMO were utilized as fluorescent molecular rotors (Figure 2a). In these luminogens, the dimethoxyl-tetraphenylethene (DMTPE), triphenylamine (TPA), and tetraphenylethene (TPE) moiety are electron donors, 
while the BMO ((Z)-4-benzylidene-2-methyloxazol-5(4H)-one) unit serves as the $\pi$-bridge and electron acceptor. This group of TICT-type AIEgens have been proved to be greatly sensitive to environmental changes such as polarity and rigidity. ${ }^{[18,39-40]}$ The absorption spectra of hexamethylene diisocyanate (HDI) solutions of these TICT-type AIEgens are similar to those of their THF solutions, showing the maximum absorption wavelength at $390 \mathrm{~nm}$ for DMTPEBMO, $410 \mathrm{~nm}$ for TPA-BMO, and $375 \mathrm{~nm}$ for TPE-BMO (Figure S1).

The fluorescence properties of the HDI solutions of TICI-type AIEgens before and after reaction with water to form polyurea (PU) solid were measured by the photoluminescence (PL) and the fluorescence quantum yields $\left(\Phi_{\mathrm{F}}\right)$. As shown in Figure $2 \mathrm{~b}$ and Figure S2a, DMTPEBMO exhibits a remarkable hypsochromic shift from orange emission with a maximum emission wavelength $\left(\lambda_{\max }\right)$ of $602 \mathrm{~nm}$ in HDI solution state to yellowish-green emission $\left(\lambda_{\max }\right.$ $=531 \mathrm{~nm}$ ) in PU solid state due to its TICT characteristic. Although DMTPE-BMO exhibits strong fluorescence in PU $\left(\Phi_{\mathrm{F}}=46 \%\right)$ because of the RIM effect, the emission intensity of its HDI solution is too weak to be distinguished $\left(\Phi_{\mathrm{F}}=2 \%\right)$. Similar to DMTPE-BMO, the fluorescence of TPA-BMO shows a 64-nm blueshift from HDI to PU (Figure 2c and Figure S2b). However, the emission intensity of TPA-BMO in the solution state $\left(\Phi_{\mathrm{F}}=68 \%\right)$ is similar to its PU state $\left(\Phi_{\mathrm{F}}=70 \%\right)$. This could be explained by the fewer rotor moieties of TPA-BMO than that of DMTPE-BMO because the change in emission intensity of fluorophores is considered to be mainly affected by intramolecular rotations. In terms of TPE-BMO, it exhibits not only a remarkable blueshift in $\lambda_{\max }$ from $556 \mathrm{~nm}$ (HDI) to $473 \mathrm{~nm}$ (PU) but also a sharp increase in fluorescent intensity with the $\Phi_{\mathrm{F}}$ increasing from $11 \%$ (HDI) to $62 \%$ (PU) (Figure $2 d$ and Figure S2c). Moreover, the fluorescence of the HDI solution of TPE-BMO can be readily detected by both fluorescence spectrophotometer and naked eyes. In other words, the HDI solution of TPE-BMO shows desired dual-fluorescence signals with significant emission 
intensity and color change after curing with water. Therefore, TPE-BMO was selected as the model optical indicator for the following study.

(a)
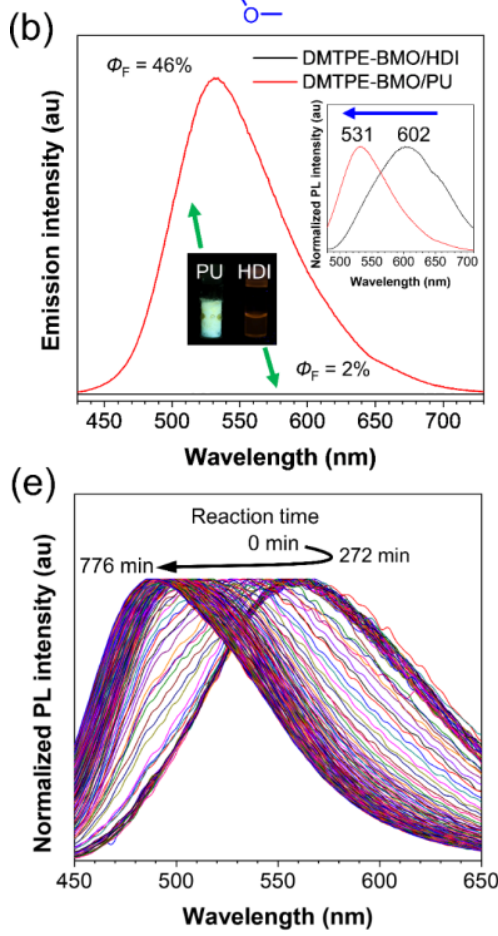

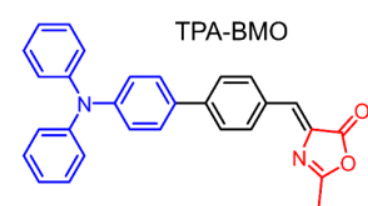

(c)
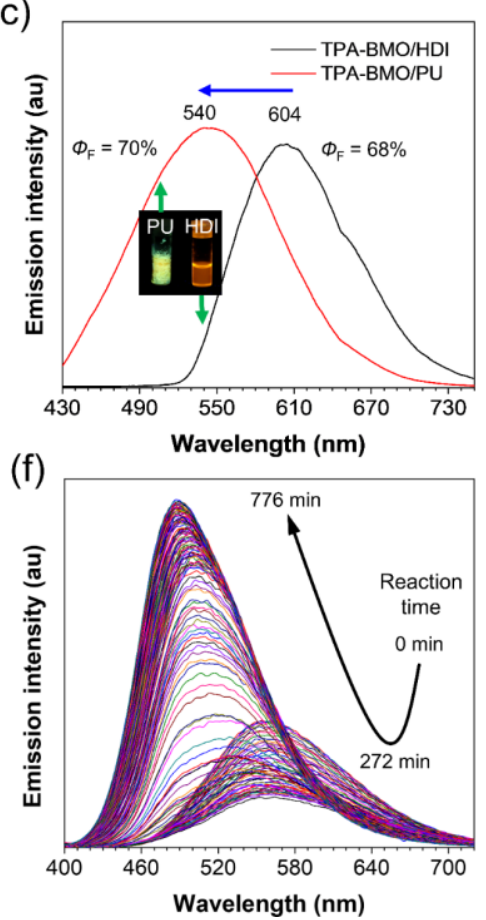

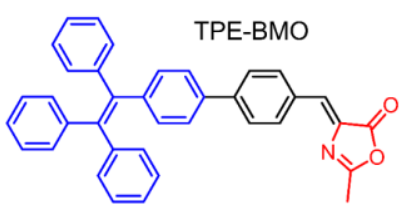

(d)
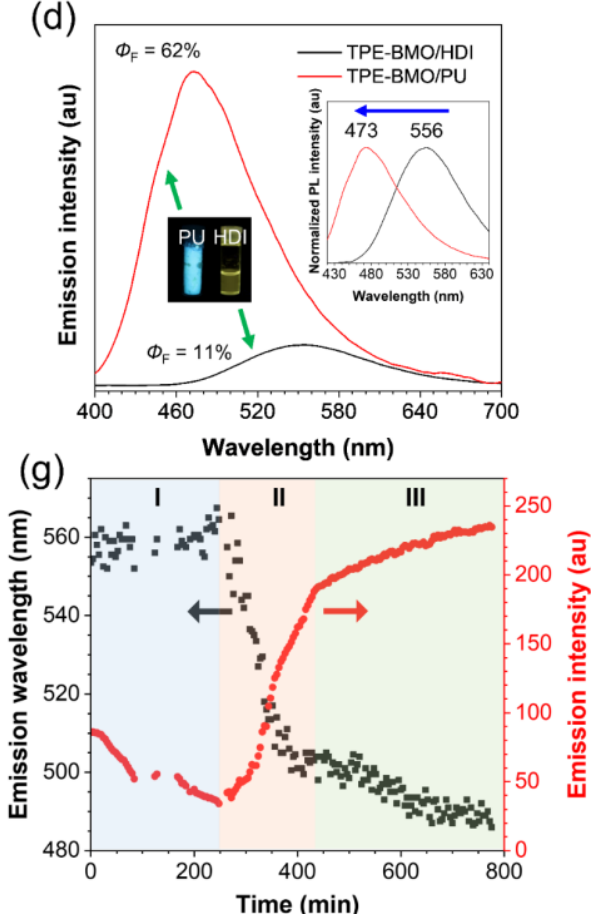

Figure 2. (a) Chemical structures of the selected TICT-type AIEgens. (b-d) PL spectra and fluorescence quantum yields ( $\Phi_{\mathrm{F}}$ ) of (b) DMTPE-BMO, (c) TPA-BMO, (d) TPE-BMO in HDI solutions and PU solids. Excitation wavelength: $390 \mathrm{~nm}$ for DMTPE-BMO; $410 \mathrm{~nm}$ for TPA$\mathrm{BMO} ; 375 \mathrm{~nm}$ for TPE-BMO. Insets are normalized PL spectra and fluorescence images of the specimens taken under $365 \mathrm{~nm}$ UV light illumination. (e and f) Time-dependent PL spectra of the TPE-BMO/HDI solution during curing reaction with water. (g) Plots of the maximum emission wavelength and emission intensity as a function of time for the TPE-BMO/HDI solution during its curing reaction with water.

To investigate the role of curing reaction on fluorescence variation, we first monitored the time-dependent PL spectra for the whole reaction process of HDI and water at $60{ }^{\circ} \mathrm{C}$ using 
fluorescence spectrophotometer. Figure 2e shows that the emission spectrum of the TPEBMO/HDI solution exhibits a first slight redshift followed by a remarkable blueshift as the reaction time goes on. The emission intensity decreases slightly at the beginning of the reaction and then increases dramatically from around $272 \mathrm{~min}$ to the end of the curing process (Figure $2 \mathrm{f}$ and Figure $\mathrm{S} 3$ ). The $\lambda_{\max }$ and maximum emission intensity were plotted as a function of time for easy comparison. As shown in Figure $2 \mathrm{~g}$, the emission wavelength shows a slight increase in the initial stage. Then a sharp decrease was observed followed by another minor decline. The variation trend of the emission intensity was exactly opposite to that of the emission wavelength. As mentioned before, TICT-type AIEgens are highly sensitive to the variation in surrounding microenvironments, such as steric restriction, polarity, and D-A efficacy and strength. ${ }^{[34-35,41]}$ Therefore, the observed variation tendency in fluorescence behaviors may arise from the competitive influence of viscosity, restriction, polarity, and temperature. Increasing viscosity or restriction is favorable for blue-shifting and enhancing the light emission, whereas the increase in microenvironment polarity and temperature tends to result in red-shifted and decreased fluorescence. In the initial stage (stage I), the increase in the viscosity of the reaction system should be very slow according to the step-growth polymerization mechanism, which allows TPE-BMO to possess good flexibility in this sol state. ${ }^{[42-44]}$ Meanwhile, the microenvironment polarity might increase due to the formation of polar units, such as $-\mathrm{NHCOOH}$ and $-\mathrm{NH}_{2}$, during the induction period of the reaction between $-\mathrm{NCO}$ groups and water (Figure 1b). The temperature was increased at this stage as the sample was gradually heated up to $60{ }^{\circ} \mathrm{C}$ in the very beginning. Besides, the exothermal effect of this curing reaction could also lead to the temperature rise of local regions. As suggested by Figure S3c, the increase in temperature can weaken and redshift the fluorescence of the TPE-BMO/HDI solution to some extent. Consequently, the unexpected slight redshift and decrease of fluorescence in the initial reaction stage could be explained by the leading role of 
microenvironment polarity and temperature variations at such circumstances. Once the crosslinking reaction occurs to transform the sol to gel state, the viscosity would increase dramatically to overwhelm the effects of polarity and temperature, thereby showing sharply increased and blue-shifted fluorescence change in the stage II. At the final stage (stage III) of the curing reaction, the intramolecular motions and the conformation of TPE-BMO molecules are restricted to a large extent. The slowly increased intensity and slightly blue-shifted emission might result from slower diffusion of monomers in the highly viscous or even solid state. ${ }^{[45]}$

Encouraged by the PL spectra results, we then explored the possibility for the direct visualization of the large-scale curing process of TPE-BMO/HDI and water based on digital image correlation (DIC). A homemade imaging system was built as shown in Figure 3a and Figure S4, which consists of a hotplate, a UV lamp controlled by a smartphone, a camera with a self-timer, and a computer program for analysis. The sample to be observed, namely a cuvette with water in the top layer and TPE-BMO/HDI in the bottom layer, was placed right under the UV lamp. To accelerate the curing reaction, the experiment was conducted at $60{ }^{\circ} \mathrm{C}$. The change in fluorescence was monitored by controllably taking photos at different time and the following post-processing of a serial of digital photographs. This measurement setup provides a simple, real-time, on-site, and continuous DIC method for the tracking of curing process. Figure $3 \mathrm{~b}$ and Figure S5 depicted the real-time fluorescence images obtained during the curing process. The HDI solution of TPE-BMO emits yellow fluorescence in its original state. After being heated up to $60{ }^{\circ} \mathrm{C}$, the yellow fluorescence becomes weak at the beginning of the reaction. Nevertheless, the fluorescence color and brightness exhibit a sharp blueshift and enhancement, respectively, as the reaction time increases from around $380 \mathrm{~min}$ to $500 \mathrm{~min}$. The fluorescence change becomes slow and small at the end of the curing reaction. It is worth noting that the fluorescence images not only can show the overall curing process in a full field of view, but also can indicate whether, when, and where the curing reaction occurs. The transition from 
liquid state to solid state observed in these digital images further supports our abovementioned explanation for the time-dependent fluorescence change. Furthermore, a computer MATLAB digitalization program was utilized to transform the fluorescence images to the average CIE color space, grayscale, and RGB channel intensity. The color chromaticity chart in Figure 3c shows that the fluorescence color changes from yellow to blue with reaction time. The blue channel intensity exhibits the biggest change compared with the green and red channel intensities (Figure S6), indicating an apparent blueshift. The time-dependent grayscale curve suggested that the emission intensity experiences a slight decline, a sharp increase, and an almost stable stage during the curing process (Figure 3d). The results obtained by the facile DIC method are nearly consistent with the PL results shown in Figure 2. 
(a)
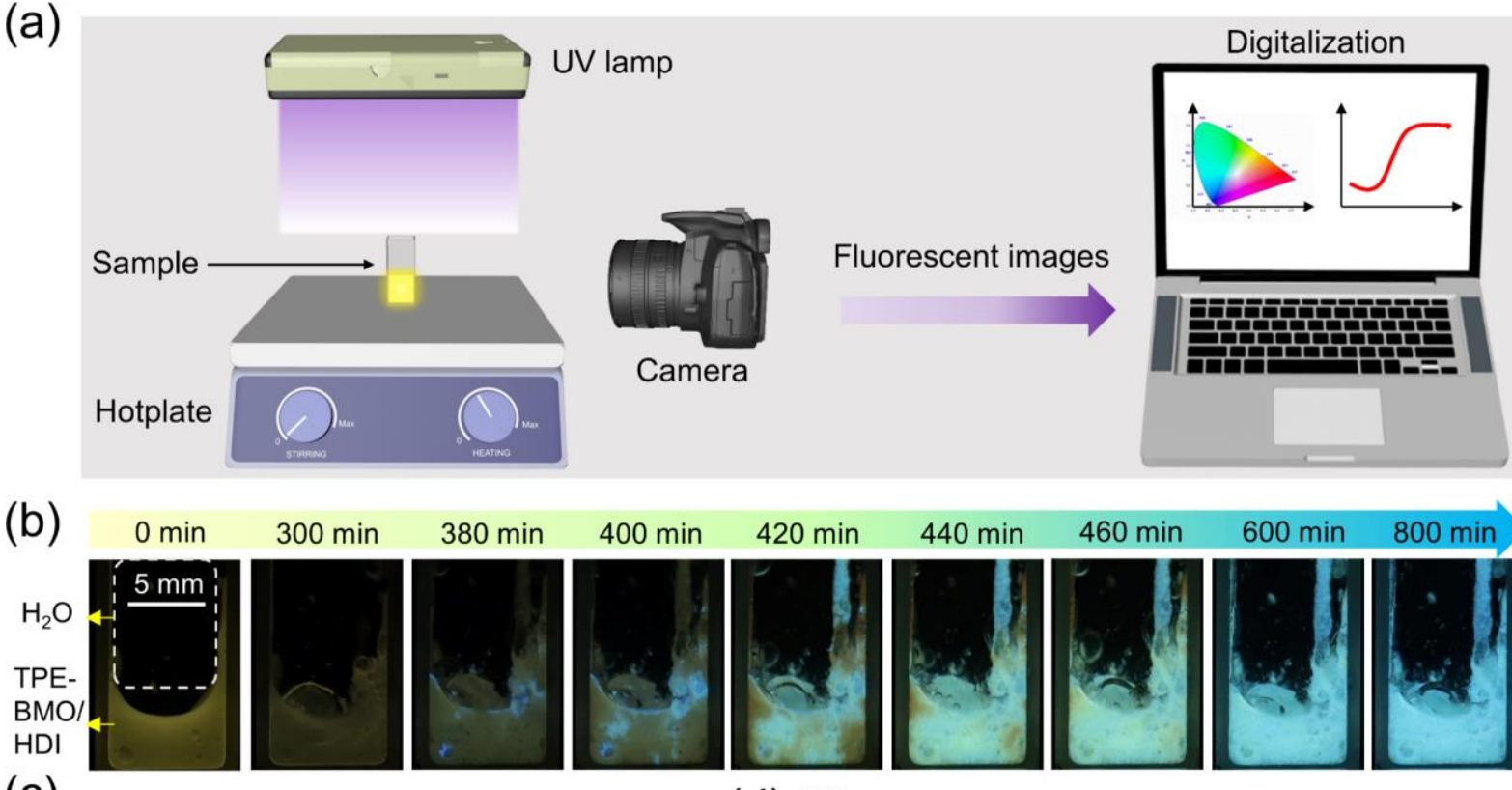

(c)
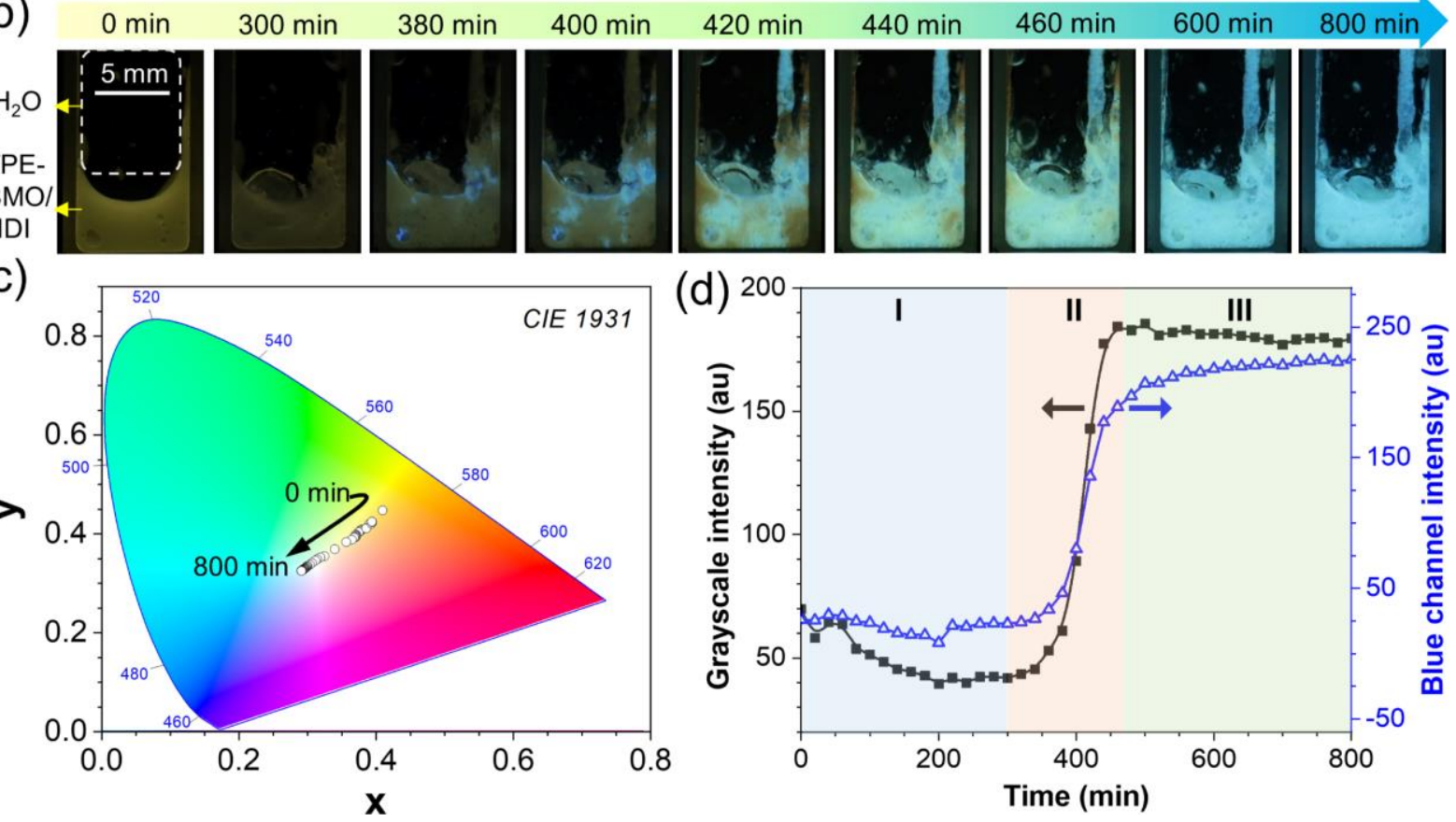

Figure 3. (a) Schematic illustration of the experimental setup for fluorescence imaging of the TPE-BMO/HDI solution during curing reaction with water. (b) Time-dependent fluorescence images of the TPE-BMO/HDI solution during curing reaction with water taken under $365 \mathrm{~nm}$ UV light illumination. (c) CIE coordinates on the CIE 1931 chromaticity diagram and (d) grayscale intensity as a function of time for the fluorescence images of the TPE-BMO/HDI solution during curing reaction with water.

To reveal the underlying working mechanism and meanwhile to verify our design principle from the mechanistic point of view, we then carried out the associated comparative experiments and theoretical calculations. Firstly, the PL spectrum of TPE-BMO/HDI solution was measured 
at $77 \mathrm{~K}$ to investigate the effect of rigidification on the fluorescence of TPE-BMO. As shown in Figure 4a, upon cooling the solution from $298 \mathrm{~K}$ to $77 \mathrm{~K}$, the relatively weak yellow emission of the TPE-BMO/HDI solution remarkably changes to bright blue emission with a 74-nm blueshift in $\lambda_{\max }$. Nevertheless, the transition from HDI solution to PU solid at room temperature led to an even more obvious blueshift of $83 \mathrm{~nm}$ in the $\lambda_{\max }$ of TPE-BMO. This experimental result suggested the synergistic effects of the rigidity and polarity of solid PU on the fluorescence behaviors of TPE-BMO, ${ }^{[46]}$ where the restriction effect might play a dominant role in fluorescence change. To gain further insight into the synergistic effects, the oscillator strength of $S_{1}$ of TPE-BMO as a function of dihedral angles was calculated (Figure $4 b$ and $4 c$ ). The results show that the oscillator strength decreases with an increase in dihedral angles. The lower is the oscillator strength, the weaker fluorescence the luminogen will show. We then calculated the optimum conformation of TPE-BMO in hexane, tetrahydrofuran (THF), and crystal state. Among them, TPE-BMO/THF was used to simulate TPE-BMO/HDI due to the similar polarity of these two solvents. ${ }^{[47]}$ The environmental polarity reduces from THF to hexane, while the restriction effect increases from THF state to crystal state. Figure S7 shows that the dihedral angle of TPE-BMO around the $\mathrm{C} 3=\mathrm{C} 4$ double bond becomes smaller when the environment polarity decreases or the restriction effect increases, thus leading to an increase in emission intensity. In this sense, the variation in fluorescence intensity from TPE-BMO/HDI to TPE-BMO/PU may be due to effects of the restriction increment and/or the polarity decline from HDI solution to PU solids. The highest occupied molecular orbital (HOMO) and the lowest unoccupied molecular orbital (LUMO) results of TPE-BMO in three states are provided in Figure 4d. The bandgap becomes wider with a decrease in polarity or an increase in restriction, which causes a hypsochromic shift in the emission spectrum. This calculation result suggests that the dramatically blue-shifted fluorescence of TPE-BMO in PU solids could result from the collective effects of the decreased polarity and the increased rigidity. 

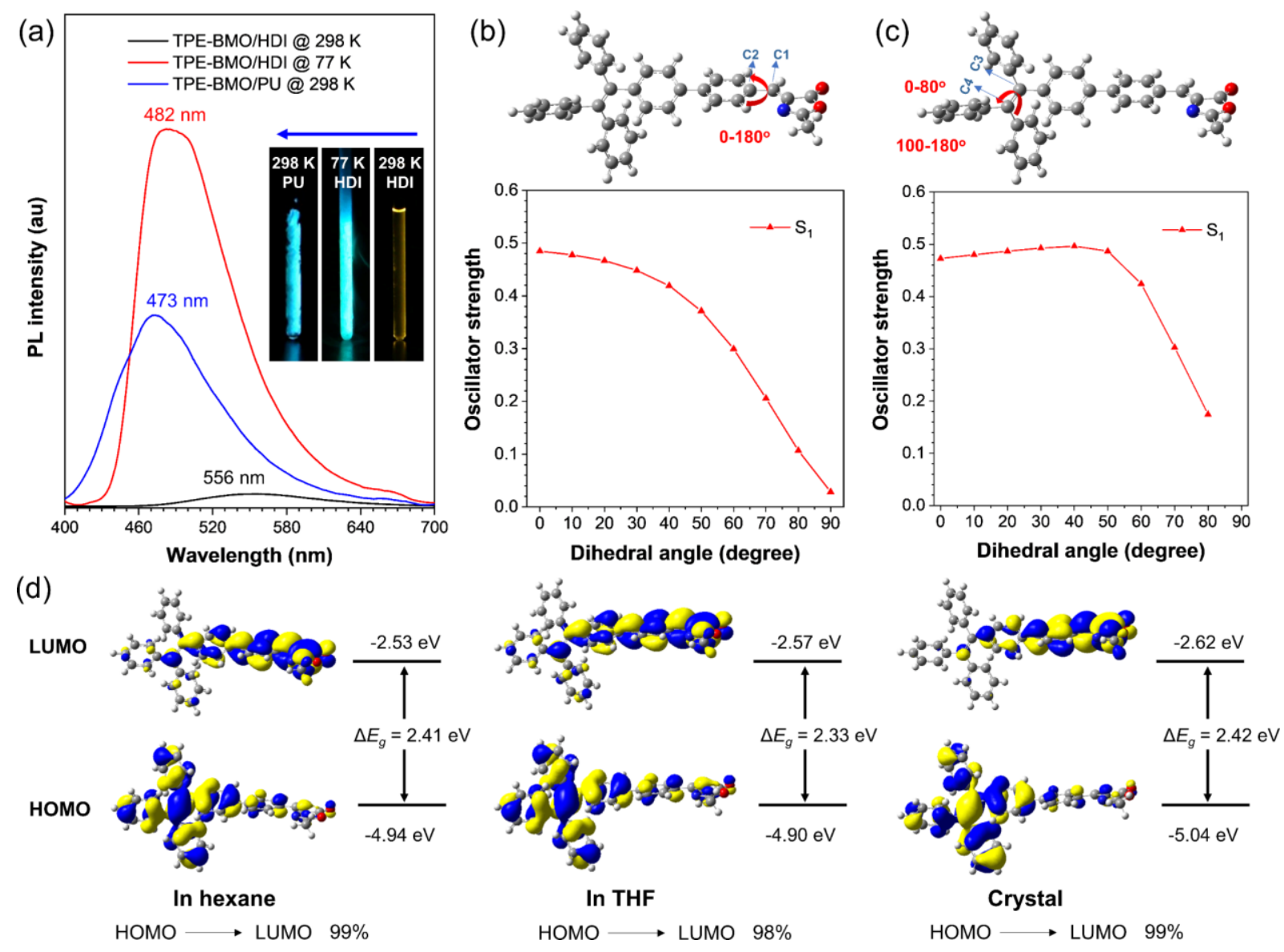

Figure 4. (a) PL spectra of TPE-BMO/HDI solutions at room temperature (298 K) and $77 \mathrm{~K}$, and TPE-BMO/PU solid at room temperature $(298 \mathrm{~K})$. Potential energy profiles of $\mathrm{S}_{1}$ and $\mathrm{S}_{0}$ with (b) rotating of $\mathrm{C} 1-\mathrm{C} 2$ single bond and (c) rotating of $\mathrm{C} 3=\mathrm{C} 4$ double bond of TPE-BMO (dihedral angle: $0-180^{\circ}$ ) in gas calculated at TD-B3LYP/6-31G(d,p) level. (d) Molecular orbitals of TPE-BMO in gas, THF (simulation for HDI), and crystal state in the transition from $\mathrm{S}_{0}$ to $\mathrm{S}_{1}$ calculated at TD-B3LYP/6-31G(d,p) level.

On the basis of the sensitive fluorescence response of TPE-BMO to microenvironment variations, we then tried to prepare the desired MCs and MC-embedded polymers to demonstrate the concept shown in Figure 1b. Novel MCs composed of UV-blocking shells and the TPE-BMO/HDI liquid core were prepared via a multi-step method. As depicted in Figure 5a, this method involved an interfacial polymerization followed by the repeated dopamine self- 
polymerization. In the first step, the oil-phase mixture of 4,4'-diphenylmethane diisocyanate (MDI) prepolymer Suprasec 2644 and TPE-BMO/HDI solution was added dropwise into the aqueous solution of polyvinyl alcohol (PVA) under agitation. Then tetraethylenepentamine (TEPA) was added into the oil-in-water emulsion to initiate the interfacial polymerization. The reaction temperature was raised to $40{ }^{\circ} \mathrm{C}$ for $3 \mathrm{~h}$ to form the single-layered MCs. Next, the obtained polyurea (PU) MCs were rinsed with deionized water for several times and then added into tris(hydroxymethyl)aminomethane (TRIS) buffer solution with a $\mathrm{pH}$ of 8.5. After adding dopamine, the mixture was stirred at room temperature for $12 \mathrm{~h}$ to deposit a layer of polydopamine (PDA) shell via the self-polymerization of dopamine. This self-polymerization process was repeated four times to deposit multiple PDA layers on the surface of MCs. The formation of four polydopamine (PDA) layers aims to improve the UV-shielding ability of MCs, thereby increasing the fluorescence contrast of intact and broken MCs. The resulting MCs of each step were denoted as PU MCs, 1PDA/PU MCs, 2PDA/PU MCs, 3PDA/PU MCs, and 4PDA/PU MCs according to the layer composition.

SEM imaging was conducted to characterize the morphologies of the MCs. Figure 5b shows that the 4PDA/PU MCs are nearly spherical, and a core-shell structure can be clearly observed. The outer surface of the MCs is rough and deposited with many small particles of PDA. Conversely, the PU MCs are too weak to keep the steric structures under a high vacuum during SEM imaging, and their outer surfaces are smooth without PDA layers (Figure S8). The composition and mechanical properties of the MCs were also characterized. As shown in Figure S9, the characteristic absorption peak of the $-\mathrm{NCO}$ group at about $2270 \mathrm{~cm}^{-1}$ can be obviously observed in the IR spectra of HDI, PU MCs and 4PDA/PU MCs, indicating the successful encapsulation of HDI. The core fraction of 4PDA/PU MCs was calculated to be around $65 \%$ according to the TGA curves, suggesting the good encapsulation efficiency of our method (Figure S10). The mechanical properties of MCs were evaluated by quasi-static 
compression at a single-capsule level. As depicted in Figure S11, the presence of PDA layer can remarkably improve the mechanical strength of the MCs compared with that of the PU MCs. Moreover, with an increase in the number of PDA layer, the nominal strength of the MCs can grow from $117 \pm 11 \mathrm{KPa}$ (1PDA/PU MCs) to $343 \pm 49 \mathrm{KPa}$ (4PDA/PU MCs).

The formation of PDA shells make the appearance color of the MCs change from yellow to black under room light, and the bright yellow fluorescence from the PU MCs can be blocked to different extent (Figure S12). When the layer number of the PDA shell increased from one to four, the observed fluorescence gradually becomes weaker and eventually become noemissive under UV illumination. To quantitatively compare the UV-shielding capability of these multi-layered MCs, a fluorescence microscope was used to detect the fluorescence of individual MCs and measure the grayscale intensity of the fluorescence images. As shown in Figure 5c, the fluorescence brightness of individual MCs gradually decreases from PU MCs to 4PDA/PU MCs under UV illumination. The corresponding grayscale value dramatically reduces from around 200 for PU MCs to 2 for 4PDA/PU MCs. These results clearly verify the excellent light-shielding ability of the 4PDA/PU shell. The fluorescence from the TPE-BMOcontaining core can be almost completely blocked after depositing four PDA layers on the MC surface. Therefore, 4PDA/PU MCs were used for the following investigation. 
(a)

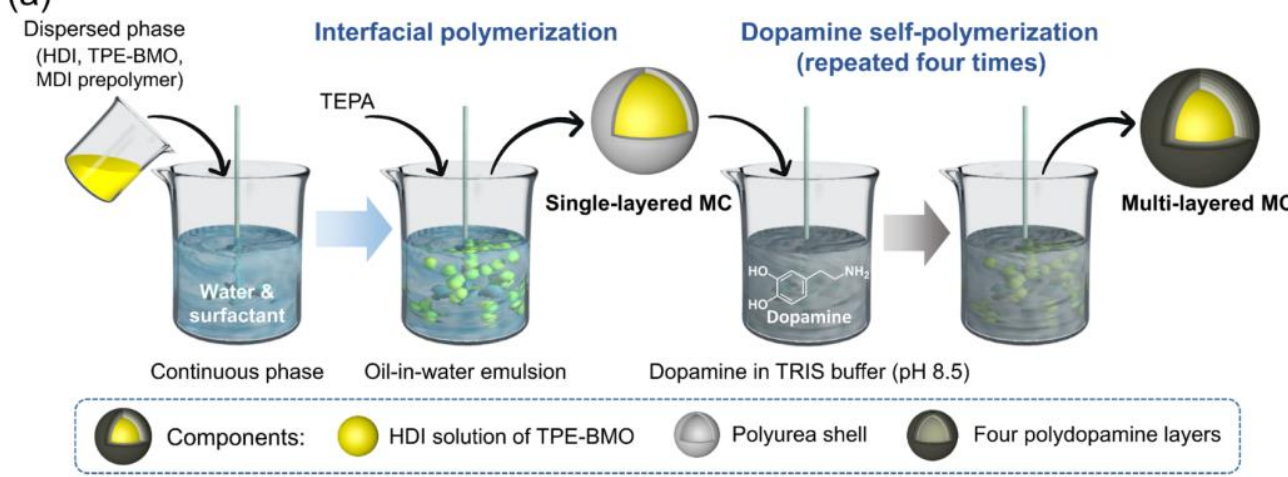

(c)

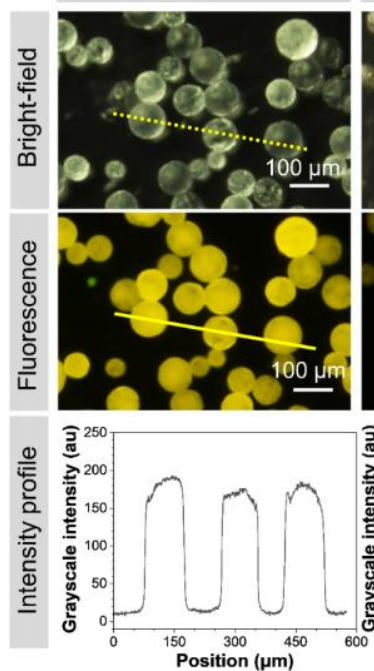

1PDAIPU MCS
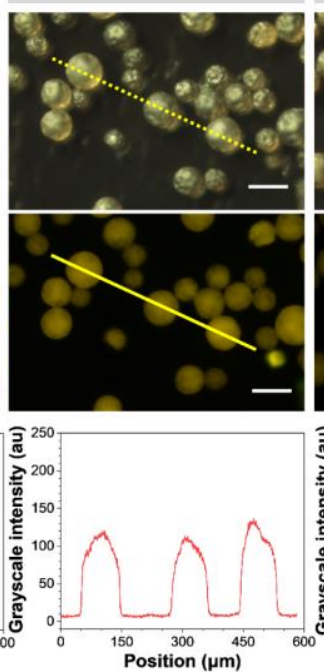

2PDAIPU MCs

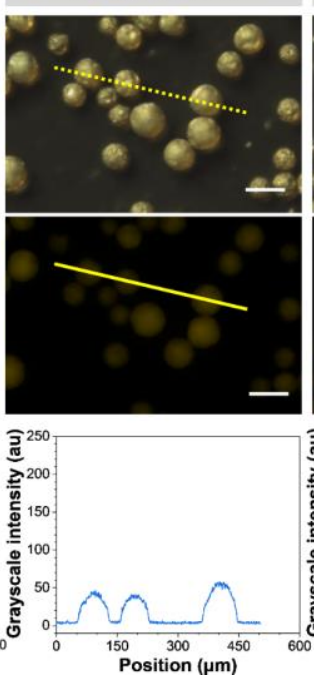

(b)
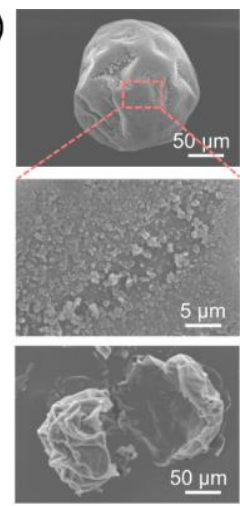

4PDAIPU MCS
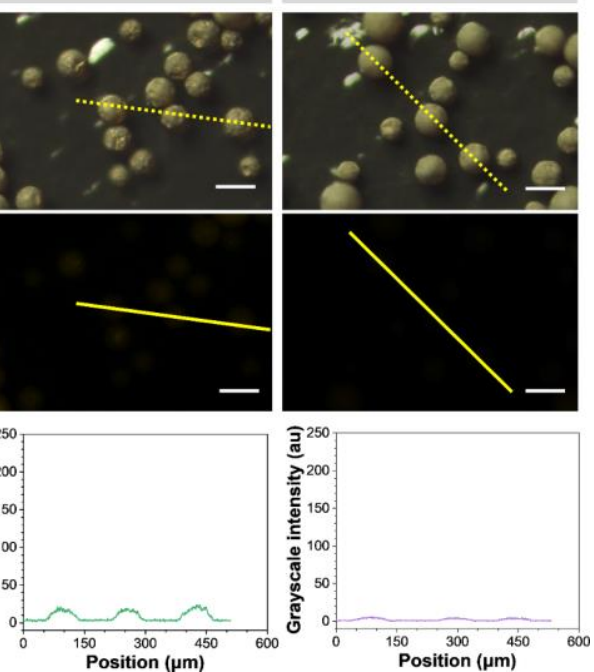

Figure 5. (a) Schematic illustration of the preparation procedures of the UV-blocking microcapsules (MCs) containing the HDI solution of TPE-BMO. (b) SEM images of 4PDA/PU MCs: an individual MC, the enlarged-outer surface, and a ruptured MC. (c) Micrographs (upper and middle panels) and the associated grayscale intensity profiles (bottom panel) of PU MCs, 1PDA/PU MCs, 2PDA/PU MCs, 3PDA/PU MCs, and 4PDA/PU MCs taken under bright field (upper panel) and the illumination of light with an excitation wavelength of 460-490 nm (middle panel). The grayscale intensity profiles correspond to the line areas in the fluorescence micrographs.

To evaluate the tracking ability of 4PDA/PU MCs containing the HDI solution of TPE-BMO (TPE-BMO/HDI MCs) for the damaging-healing process, a few intact and damaged MCs were simultaneously spread on a glass slide and observed by a fluorescence microscope. As shown in Figure 6a, the intact MCs are non-emissive, whereas the damaged MCs emit yellow fluorescence at the beginning. The emission color gradually blue-shifts with reaction time. 
Apart from the blueshift of fluorescence, an increase in the emission intensity was observed as well, which indicates the potential of TPE-BMO/HDI MCs for the visualization of the damagehealing process with dual-fluorescence signals. To investigate the visualization application of TPE-BMO MCs in polymer coatings, a MC-embedded epoxy (EP) block was prepared by casting in a cylindrical vessel and then demoulding. As presented in Figure $6 \mathrm{~b}$ and Figure S13, no fluorescence can be observed for the intact specimen under UV illumination. Upon being manually scratched, the injured regions can be readily indicated by the immediate occurrence of bright yellow luminescence. By contrast, it is difficult to distinguish the damaged areas of the sample under room light. This result confirmed the powerful damage-reporting capability of the fluorescence-based sensing technique. As the reaction time increases, the evolution of the entire healing process can be clearly monitored in a large field of view by an obvious variation in both the fluorescence color and the fluorescence intensity. The damaged regions steadily changes from the relatively weak yellow fluorescence $(0 \mathrm{~h})$ to strong blue emission $(9$ h) at room temperature. Moreover, accidental and minor damage (inside the gray frames of Figure $6 \mathrm{~b}$ ) of the EP plate that is hard to observe at the very beginning can also be repaired and obviously indicated after the self-healing process. It is worth noting that no emission can be observed from intact MCs during the whole observation period, suggesting the excellent stability of the MCs as well as the advantages of high contrast and low background noise of this visualization method in practical applications. In addition, the fluorescence intensity of these fluorescence images can be transformed to grayscale by the facile DIC method using MATLAB program, and the whole EP sample can be mapped with a color bar ranging from blue to red (Figure S14). All these results demonstrated that the proposed method is promising to be used for the direct, real-time, on-site, full-field, and sensitive visualization and monitoring of damaging-healing processes of polymers. 
(a)

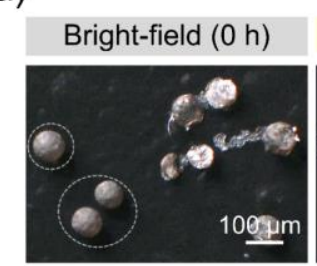

(b)

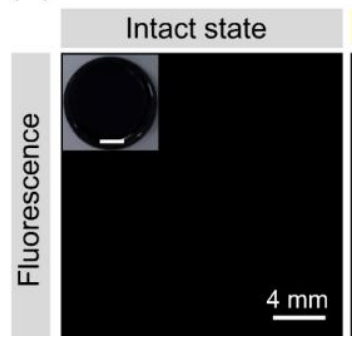

TPE-BMO/HDI MCs

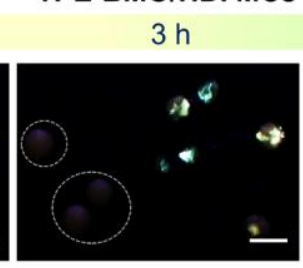

MC-embedded EP

$3 \mathrm{~h}$

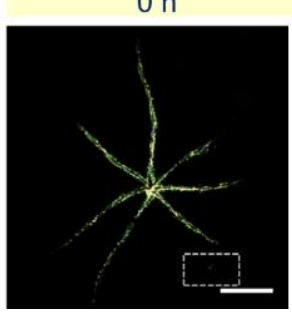

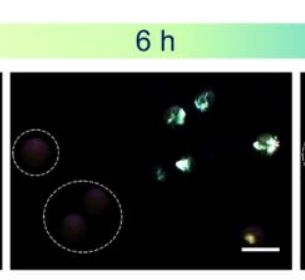
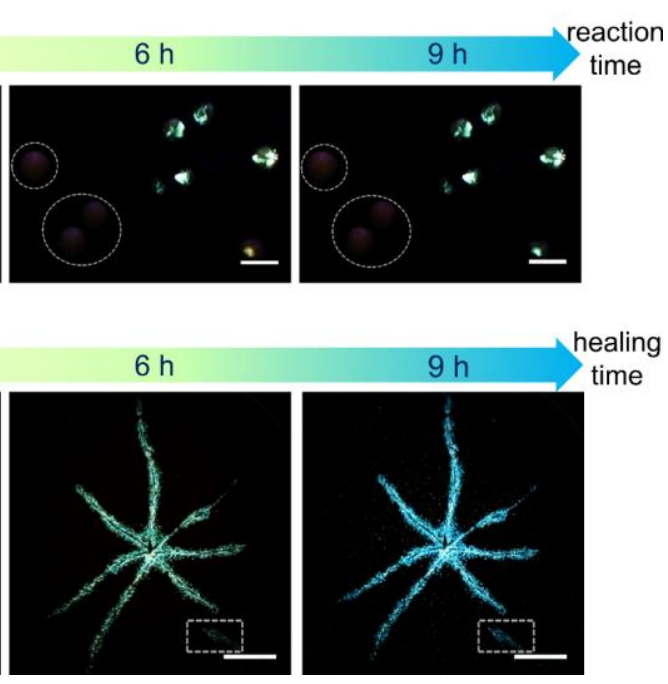

Figure 6. (a) Micrographs of 4PDA/PU microcapsules containing the HDI solution of TPEBMO (TPE-BMO/HDI MCs) taken under bright field and $365 \mathrm{~nm}$ UV light illumination at different reaction time during the healing process. (b) Fluorescent images of the MC-embedded epoxy (EP) coating in the intact state and damaged state at different healing time. The inset is the whole photograph of the intact EP coating taken under room light (scale bar $=8 \mathrm{~mm}$ ).

A correlation between our fluorescence-based sensing method and the traditional selfhealing characterization techniques was then investigated. A pure EP coating and the MCembedded EP coatings was coated on aluminized paper, respectively, and narrow throughthickness cracks were created with a sharp blade (Figure 7a). The cracks of the MC-embedded EP coatings initially show weak yellow emission and then gradually emit bright-blue fluorescence over time, whereas no signal was detected from the pure EP coating. The SEM images reveal that the cracks of MC-embedded EP coatings are partially sealed without sufficient healing time such as at $3 \mathrm{~h}$ and $6 \mathrm{~h}$. After healing for $9 \mathrm{~h}$, the cracks were completely filled by the newly formed materials. These results are consistent with the variation in fluorescence signals. Compared with the SEM technique, our method possesses the unique onsite and full-field characteristics for complicated real sample. To further check the health state of the cracked polymer coatings, a water permeation test was performed using an experiment 
setup shown in Figure $7 b .{ }^{[48]}$ Figure $7 \mathrm{c}$ shows that the pure EP coating underwent severe water permeation. In terms of the MC-embedded EP coatings, the average weight of permeated water decreased from $5.5 \mathrm{~g}$ for the unhealed sample to $2.9 \mathrm{~g}$ and $1.3 \mathrm{~g}$ for the partially healed ones. No water permeation was observed for the fully-healed specimen. This result indicates that the fluorescence signals of the MC-embedded coatings can be directly correlated to their health states such as the water-proof degree.

(a) Pure EP coating
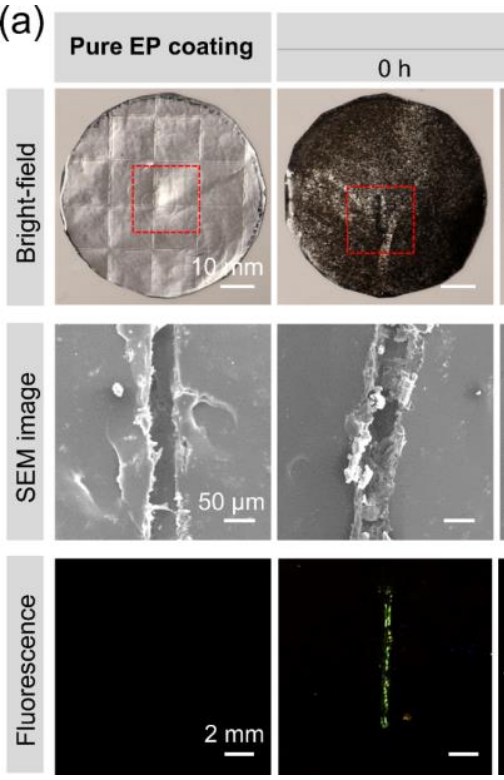

MC-embedded EP coating
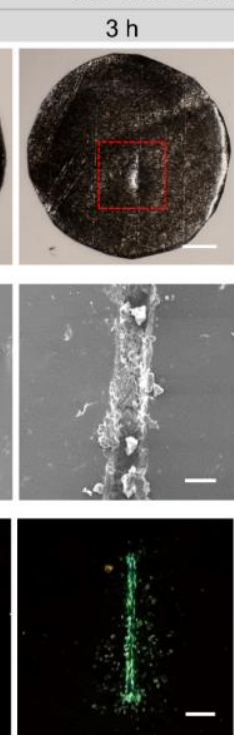

$6 \mathrm{~h}$
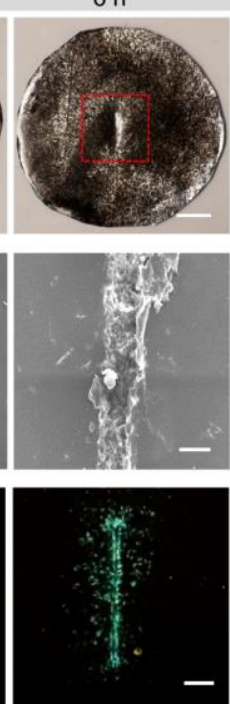

(b)

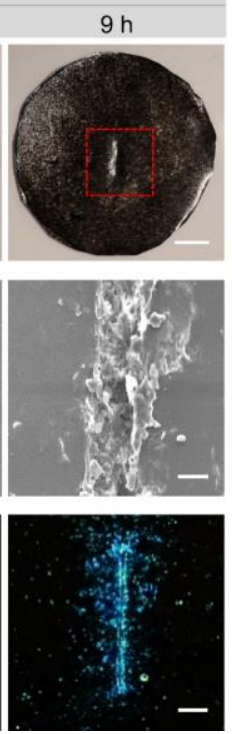

(c)
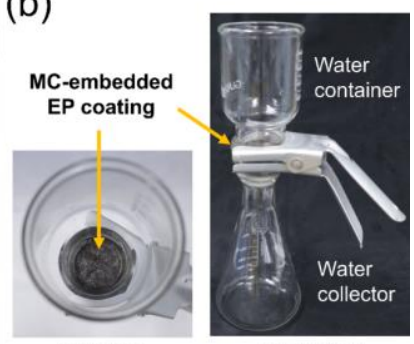

Front view

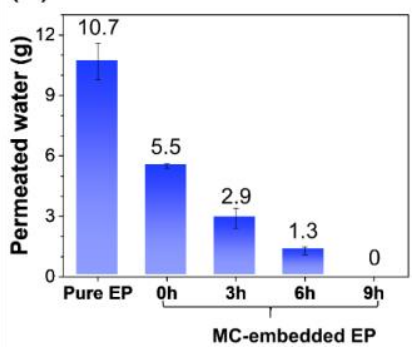

Figure 7. (a) Bright-field photographs, SEM images, and fluorescence images of a pure EP coating and the MC-embedded EP coatings at different healing time. (b) Experiment setup of water permeation test. (c) The water-permeation capability of the cracked pure EP coating and MC-embedded EP coatings at different healing time.

Taking advantage of the light-shielding ability of the MC shell, we further investigated the photoprotection property of the MC-embedded EP coating. As shown in Figure 8a, due to the Tyndall effect, a light path from a red laser pointer pen can be clearly observed in a bottle of silica sol without the polymer coating. In sharp contrast, the Tyndall phenomenon disappears after placing an MC-embedded EP coating in front of the silica sol. The optical transmittance of the MC-embedded EP coating on quartz glass was also measured and compared with that of 
a pure EP coating. As depicted in Figure 8b, the pure EP coating exhibits high transmittances of about $84 \%$ in the visible light region (400-800 nm) and above 50\% in the UV light region of 350-400 nm. By comparison, the MC-embedded EP coating shows transmittances of lower than $3 \%$ for the whole testing wavelength range. These results convincingly demonstrate the excellent photoprotection performance of the MC-embedded coating.

Considering the autonomous damage-healing indication capability, excellent UV-Vis lightshielding property, as well as the direct and sensitive visualization of water-proof degree, of the MC-embedded EP composites are promising to serve as multifunctional coating materials in packaging applications for the storage of photo- and/or water-sensitive objects, such as photoresist, photosensitizers, and electronic memory chips. To demonstrate the applicability of such EP coatings, we then conducted a proof-of-concept study. As depicted in Figure 8c, a pure EP coating and a MC-embedded EP coating was covered on the front side of thin plastic boxes containing photosensitive powders, respectively. After placing the boxes under sunlight illumination for $5 \mathrm{~min}$, the color of the photosensitive powders in the pure EP-coated box remarkably changes from white to orange, while those in the box coated with MC-embedded EP keeps stable with little color change. In addition to the photoprotection function, the damage-warning, health indication, self-healing, and water-proof functionalities of such coatings were also demonstrated. As shown in Figure 8d-f, when narrow through-thickness cracks were caused to the coatings, these cracks were hardly observed under room light or in pure EP coating but can be sensitively indicated in the MC-embedded EP coating under UV light illumination. After immersing the coated boxes underwater and then placing them on a hotplate to evaporate the inside permeated water, lots of moisture was observed from the back side of the EP-coated box (lower panel of Figure 8f). On the contrary, the boxes coated with MC-embedded EP coatings exhibit less or even no moisture inside due to the reaction of the released HDI with water. Moreover, the whole healing process can be readily monitored by the 
dual-fluorescence variations. In other words, users could evaluate the health state or the waterproof performance of the functional coatings simply by fluorescence signals in a real-time and on-site way. In this regard, this method could help the related users to take necessary actions before the malfunction or failure occurs. Overall, the MC-embedded EP coating can protect the photosensitive components, retard the water permeation through the damaged containers, and visually assess the water-proof degree, all of which are desirable functionalities for practical packaging applications.

(a)

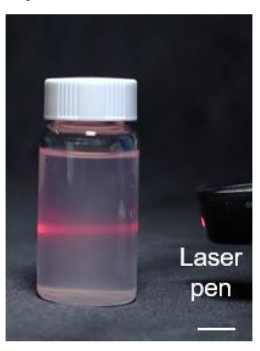

(d)

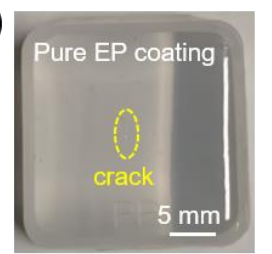

(e)

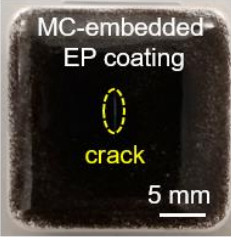

(b)

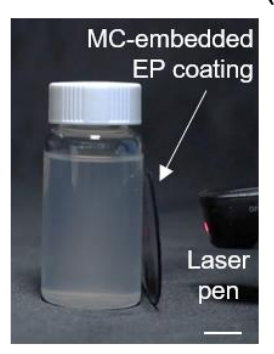

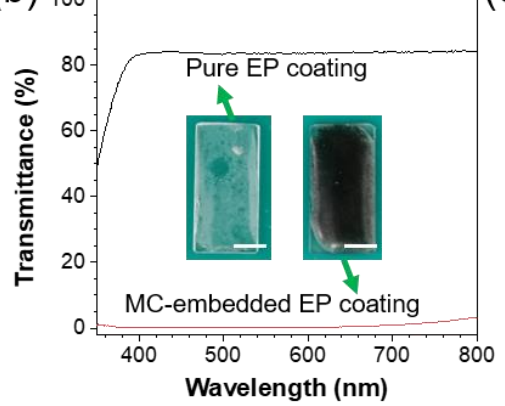

(c)
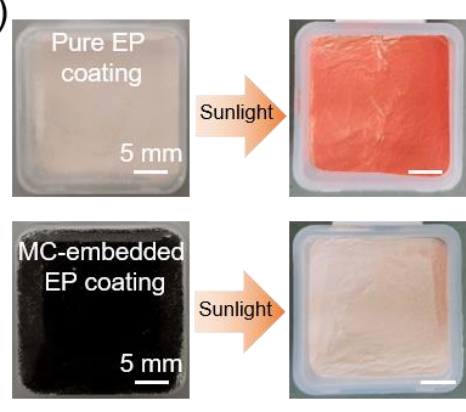

(f)

Pure EP coating

$\mathrm{Oh}$

MC-embedded EP coating

$3 \mathrm{~h}$

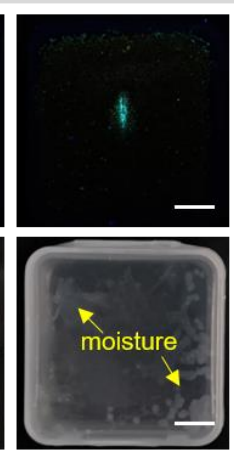

$6 \mathrm{~h}$

$9 \mathrm{~h}$

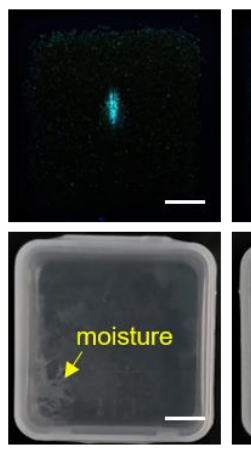

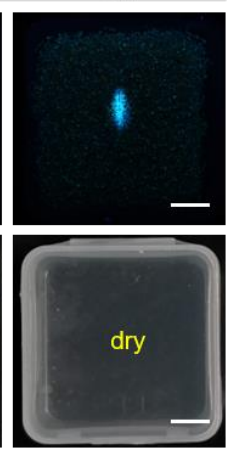

Figure 8. (a) The Tyndall effect in a bottle of silica sol without and with an MC-embedded EP. The scale bar indicates $2 \mathrm{~mm}$. (b) UV-Vis transmittance spectra and the associated photographs of quartz glass plates coated with a pure EP coating and a MC-embedded EP coating, respectively. The scale bar in the insets indicates $5 \mathrm{~mm}$. (c) Photographs showing the front sides of boxes coated with pure EP and MC-embedded EP, and their inside photosensitive powders before and after placing under sunlight for 5 min. (d and e) Photographs showing the front side of a cracked box coated with (d) pure EP coating and (e) MC-embedded EP coating 
taken under room light. (f) Fluorescence images showing front sides of cracked boxes coated with pure EP coating or MC-embedded EP coatings at different healing time (upper panel). Bright-field photographs showing back sides of cracked boxes with pure EP coating and MCembedded EP coating at different healing time after evaporating the penetrated water inside (lower panel).

In conclusion, this work provided a simple yet powerful fluorescence-based method to visualize and monitor the damaging-healing processes of MC-based self-healing polymer coatings, in which a TICT-type AIEgen (TPE-BMO) was used as the optical indicator. Due to the collective effects of AIE and TICT, TPE-BMO exhibits dual signal changes in both fluorescence intensity and fluorescence color in response to microenvironment variations of its HDI solution. Such a sensitive fluorescence response can be detected by naked eyes, fluorescence spectrometer, and DIC analysis. By encapsulating the HDI solution of TPE-BMO inside UV-blocking shells, robust and multifunctional MCs with light-shielding and damagehealing indication capabilities were prepared. The dispersion of the AIEgen-loaded MCs in EP matrix produced polymer coatings with damage-reporting, autonomous self-healing, and health indication functionalities. Immediate turn-on fluorescence was observed upon the composite coatings were damaged, and the fluorescence in the damaged areas gradually blue-shifted and enhanced with the healing time. Furthermore, the smart polymer coatings exhibit excellent photo- and water-protective performance. Considering these attractive properties, the MCembedded polymer composites are promising to serve as multifunctional coating materials in packaging applications. Compared with traditional characterization methods, this fluorescencebased method can achieve the direct and full-field visualization and monitoring of damaginghealing processes of polymers in a simple, non-destructive, real-time, and on-site way. It is envisioned that such a versatile and multifunctional visualization method with a clear working mechanism can be extended to other self-healing systems and will open up new opportunities 
for numerous applications of smart coatings in the packaging, automobile, and shipping industries.

\section{Supporting Information}

Supporting Information is available from the Wiley Online Library or from the author.

\section{Acknowledgment}

The work was financially supported by the National Natural Science Foundation of China (Grant \#: 21905176), the Project of Hetao Shenzhen-Hong Kong Science and Technology Innovation Cooperation Zone (HZQB-KCZYB-2020083), the NSFC/RGC Joint Research Scheme of Hong Kong (Grant\#: N_HKUST 631/18, 51861165103), and the Science and Technology Plan of Shenzhen (JCYJ20190808142403590). The authors are grateful to Mark Ellwood from MAE of HKUST for the discussion of this work. The authors also acknowledge the Instrumental Analysis Center of Shenzhen University.

\section{Conflict of Interest}

The authors declare no conflict of interest.

\section{References}

[1] J. F. Patrick, M. J. Robb, N. R. Sottos, J. S. Moore, S. R. White, Nature 2016, 540, 363.

[2] M. Scheiner, T. J. Dickens, O. Okoli, Polymer 2016, 83, 260.

[3] A. K. Leonardi, C. K. Ober, Annual review of chemical and biomolecular engineering 2019, 10, 241.

[4] J. J. Licari, Coating materials for electronic applications: polymers, processing, reliability, testing, William Andrew Publishing, Norwich, NY 2003.

[5] O. Rifaie-Graham, E. A. Apebende, L. K. Bast, N. Bruns, Advanced Materials 2018, 30, 1705483.

[6] S. McDonald, S. Coban, N. Sottos, P. Withers, Scientific reports 2019, 9, 1.

[7] L. Reimer, Ultramicroscopy 1984, 14, 291.

[8] S. Biria, I. D. Hosein, Macromolecules 2017, 50, 3617. 
[9] S. K. Dwivedi, M. Vishwakarma, A. Soni, Materials Today: Proceedings 2018, 5, 3690.

[10] M. M. Caruso, D. A. Davis, Q. Shen, S. A. Odom, N. R. Sottos, S. R. White, J. S. Moore, Chemical reviews 2009, 109, 5755.

[11] D. Y. Zhu, M. Z. Rong, M. Q. Zhang, Progress in Polymer Science 2015, 49, 175.

[12] C. E. Diesendruck, N. R. Sottos, J. S. Moore, S. R. White, Angewandte Chemie International Edition 2015, 54, 10428.

[13] D. B. Lioi, V. Varshney, S. Izor, G. Neher, W. J. Kennedy, Journal of Materials Chemistry C 2019, 7, 14471.

[14] R. Kotani, H. Sotome, H. Okajima, S. Yokoyama, Y. Nakaike, A. Kashiwagi, C. Mori, Y. Nakada, S. Yamaguchi, A. Osuka, Journal of Materials Chemistry C 2017, 5, 5248.

[15] J. B. Birks, Photophysics of aromatic molecules, Wiley, London 1970.

[16] J. Mei, N. L. Leung, R. T. Kwok, J. W. Lam, B. Z. Tang, Chemical reviews 2015, 115, 11718.

[17] Z. Wang, J. Nie, W. Qin, Q. Hu, B. Z. Tang, Nature communications 2016, 7, 1.

[18] T. Han, C. Gui, J. W. Lam, M. Jiang, N. Xie, R. T. Kwok, B. Z. Tang, Macromolecules 2017, 50, 5807.

[19] M. Gao, Y. Cheng, J. Zhang, C. Xu, X. Yu, M. Zhu, Chemical Research in Chinese Universities 2021, 1.

[20] Q. Li, K. Huang, Q. Qiu, X. Zhang, D. Qin, X. Zeng, Dyes and Pigments 2021, 109537.

[21] M. Adeel, B. Zhao, S. Xu, S. Zheng, The Journal of Physical Chemistry B 2019, 123, 6282.

[22] T. Han, L. Liu, D. Wang, J. Yang, B. Z. Tang, Macromolecular Rapid Communications 2021, 42, 2000311.

[23] A. Funtan, P. Michael, S. Rost, J. Omeis, K. Lienert, W. H. Binder, Advanced Materials 2021, 33, 2100068.

[24] M. J. Robb, W. Li, R. C. Gergely, C. C. Matthews, S. R. White, N. R. Sottos, J. S. Moore, ACS central science 2016, 2, 598.

[25] C. Calvino, A. Guha, C. Weder, S. Schrettl, Advanced Materials 2018, 30, 1704603.

[26] C. Calvino, E. Henriet, L. F. Muff, S. Schrettl, C. Weder, Macromolecular rapid communications 2020, 41, 1900654.

[27] X. Lu, W. Li, N. R. Sottos, J. S. Moore, ACS applied materials \& interfaces 2018, 10, 40361.

[28] Y. K. Song, B. Kim, T. H. Lee, J. C. Kim, J. H. Nam, S. M. Noh, Y. I. Park, Macromolecular rapid communications 2017, 38, 1600657.

[29] S. Chen, T. Han, Y. Zhao, W. Luo, Z. Zhang, H. Su, B. Z. Tang, J. Yang, ACS applied materials \& interfaces 2019, 12, 4870.

[30] Y. K. Song, T. H. Lee, K. C. Lee, M. H. Choi, J. C. Kim, S.-H. Lee, S. M. Noh, Y. I. Park, Applied Surface Science 2020, 511, 145556.

[31] Y. K. Song, T. H. Lee, J. C. Kim, K. C. Lee, S.-H. Lee, S. M. Noh, Y. I. Park, Molecules 2019, 24, 1679.

[32] H. Qian, M. E. Cousins, E. H. Horak, A. Wakefield, M. D. Liptak, I. Aprahamian, Nature chemistry 2017, 9, 83.

[33] Y. Cheng, J. Wang, Z. Qiu, X. Zheng, N. L. Leung, J. W. Lam, B. Z. Tang, Advanced Materials 2017, 29, 1703900.

[34] Z. R. Grabowski, K. Rotkiewicz, W. Rettig, Chemical reviews 2003, 103, 3899.

[35] S. Sasaki, G. P. Drummen, G.-i. Konishi, Journal of Materials Chemistry C 2016, 4, 2731.

[36] C. Peinado, E. Salvador, F. Catalina, A. Lozano, Polymer 2001, 42, 2815.

[37] J. Yang, M. W. Keller, J. S. Moore, S. R. White, N. R. Sottos, Macromolecules 2008, 41,9650 . 
[38] M. Huang, J. Yang, Journal of Materials Chemistry 2011, 21, 11123.

[39] Y. Zhang, M. Jiang, T. Han, X. Xiao, W. Chen, L. Wang, K. S. Wong, R. Wang, K. Wang, B. Z. Tang, ACS applied materials \& interfaces 2018, 10, 34418.

[40] M. Jiang, Z. He, Y. Zhang, H. H. Sung, J. W. Lam, Q. Peng, Y. Yan, K. S. Wong, I. D. Williams, Y. Zhao, Journal of Materials Chemistry C 2017, 5, 7191.

[41] D. Liese, G. Haberhauer, Israel Journal of Chemistry 2018, 58, 813.

[42] G. Yilmaz, Y. Yagci, Progress in Polymer Science 2020, 100, 101178.

[43] S. Ye, H. Zhang, J. Fei, C. H. Wolstenholme, X. Zhang, Angewandte Chemie International Edition 2021, 60, 1339.

[44] R. Hu, E. Lager, A. Aguilar-Aguilar, J. Liu, J. W. Lam, H. H. Sung, I. D. Williams, Y. Zhong, K. S. Wong, E. Pena-Cabrera, The Journal of Physical Chemistry C 2009, 113, 15845.

[45] H. Ni, H. A. Nash, J. G. Worden, M. D. Soucek, Journal of Polymer Science Part A: Polymer Chemistry 2002, 40, 1677.

[46] Y. Hu, L. Barbier, Z. Li, X. Ji, H. Le Blay, D. Hourdet, N. Sanson, J. W. Lam, A. Marcellan, B. Z. Tang, Advanced Materials 2021, 33, 2101500.

[47] Y. Zhang, M. Jiang, G.-C. Han, K. Zhao, B. Z. Tang, K. S. Wong, The Journal of Physical Chemistry C 2015, 119, 27630.

[48] H. Zhang, Y. B. Chong, Y. Zhao, A. Buryak, F. Duan, Engineered Science 2019, 8, 66. 